\title{
Car Theft Identification, Tracking and Control System
}

\author{
${ }^{1}$ Nithin Kurup U.G (M.Tech), ${ }^{2}$ G.Vara Lakshmi \\ ${ }^{I}$ Computer Science and Engineering Aurora's Technological and Research Institute Parvathapur, Uppal, \\ Hyderabad, India \\ ${ }^{2}$ Associate Professor Aurora's Technological and Research Institute Parvathapur, Uppal, Hyderabad, India
}

\begin{abstract}
This paper proposes an integrated system consisting of a GPS (Global positioning System) module, an Android mobile phone. and a Control system to be used in cars for theft Identification, tracking and controlling.PCA (Principal Component Analysis) is used for FRS(Face Recognition System). If the car seems to be theft, the owner will be conveyed with image of the thief and latitude and longitude information through MMS (Multimedia Messaging Service) and SMS (Short Messaging Service). The Short Messaging Service will send to owner's mobile periodically and on receiving the SMS the owner has to send a secret code through SMS to the car, then the car will stop immediately; for that a Microcontroller has to be placed in the car which will stop the car by using the relay system in the car. This is cheaper and reliable one.
\end{abstract}

Keywords- Global positioning System, Android mobile phone, Control system, Principal Component Analysis, Multimedia Messaging Service, Short Messaging Service.

\section{Introduction}

Today car security is one of the challenging issues in our society. Biometric recognition, image processing, communication etc are the currently using techniques. All these security system using many sensors, so the cost of implementation is high.

The car consists of a tiny digital camera, GPS(Global Positioning System) module, a GSM(Global System for Mobile Communication) module and a control system .Control system mainly consists of a microcontroller, RS-232(Recommended Standard-232) Cables ,MAX232 IC,power supply. The PCA (Principal Component Analysis) algorithm have been first profiled in MATLAB (Matrix laboratory) and then implemented on ANDROID phone.

When car is theft by somebody then the FRS (Face Recognition System) obtains face images by tiny digital camera which can hide easily somewhere in the car.PCA will matches the captured face image with library of known faces. Known faces means already some of the face images will be store in Android phone like owners face image If the face images are not matched then a MMS (Multimedia Messaging Service), SMS(Short Messaging Service) will be sent to owners mobile.MMS contains the captured face image nothing but thief image.SMS contains the latitude and longitude information of car.SMS will be sent from car periodically to owners mobile. Once the owner is sends secret code through SMS the car will stop immediately and the SMS sending from the car to owner's mobile will be throttle down. The integer values are used for secret code.

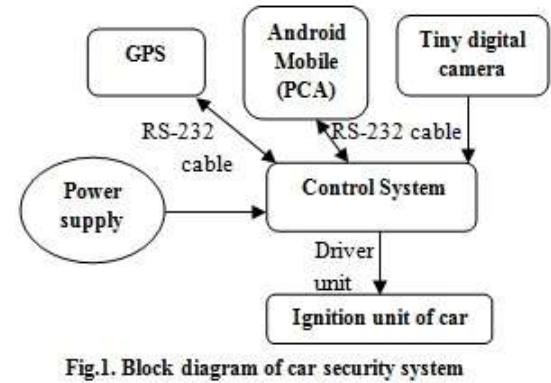

Fig.1. Block diagram of car security system

All steps are controlled by the microcontroller in control system, including capturing images, face recognition, getting GPS information, sending MMS, SMS, stopping the car.

\section{Face Recognition}

Face recognition algorithms are used in a wide range of applications viz., security control, crime investigation, and entrance control in buildings, access control at automatic teller machines, passport verification, identifying the faces in a given databases. 
The Principal Component Analysis (PCA) approach is also known as Eigen faces. PCA is a useful statistical technique that has found application in fields such as face recognition and image compression, and is a common technique for finding patterns in data of high dimension. The basic goal is to implement a simple face recognition system, based on well-studied and well-understood methods. One can choose to go into depth of one and only one of those methods. The method to be implemented is PCA(Principle Component Analysis). It is one of the most successful techniques of face recognition and easy to understand and describe using mathematics. This method involves the Eigen faces.

In PCA the first step is to produce a feature detector (dimension reduction).Principal Components Analysis (PCA) was chosen because it is the most efficient technique, of dimension reduction, in terms of data compression. This allows the high dimension data, the images, to be represented by lower dimension data and so hopefully reducing the complexity of grouping the images

\subsection{Methodology}

\subsubsection{Data Acquisition}

A database of different image sets of faces was constructed. It's only got 2 dimensions, and the reason why one can have chosen this is so that one can provide plots of the data to show what the PCA analysis is doing at each step.

\subsection{Subtract the mean image data}

For PCA to work properly, you have to subtract the mean from each of the data dimensions. The mean subtracted is the average across each dimension. So, all the $\mathrm{x}$ values have $\mathrm{x}$ ' (the mean of the $\mathrm{x}$ values of all the data points) subtracted, and all the y values have y' subtracted from them. This produces a data set whose mean is zero.

\subsection{Find the covariance matrix}

Covariance is always measured between 2 dimensions. The data is 2 dimensional the covariance matrix will be $2 * 2$ as shown :

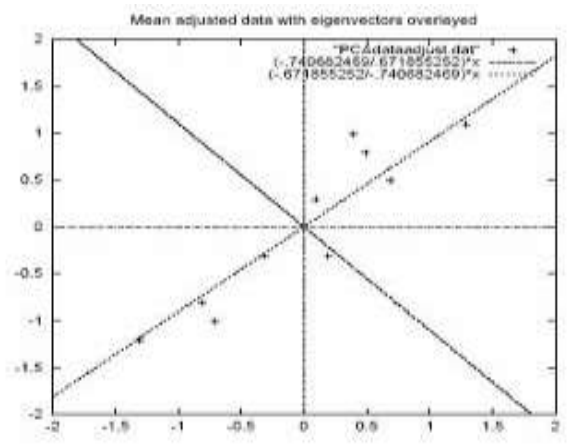

Fig.2: A plot of the normalized data (mean Subtracted) with the Eigen vectors of the covariance matrix.

\subsubsection{Face Recognition Process in Eigen faces Approach.}

Step 1. Build a face database that consists of the face images of car owner and relatives.

Step 2. Choose a training set that includes a number of images (M) for each person with some variation in pose and different faces.

Step 3. Calculate the $M \times M$ matrix L, find its Eigen vectors and Eigen values, and choose the M' Eigen vectors with the highest associated Eigen values..

Step 4. Combine the normalized training set of images to produce M' Eigen faces.

Step 5. Store these Eigen faces for later use.

Step 6. For each member in the face database, compute and store a feature vector.

Step 7. Choose a threshold value e that defines the maximum allowable distance from any face class. Optionally choose a threshold $f$ that defines the maximum allowable distance from face space.

Step 8. For each new face image i.e. images captured by tiny digital camera in car to be identified, calculate its feature vector and compare it with the stored feature vectors of the face library members.

Step 9. If the comparison satisfies the threshold for at least one member, then classify this face image as "known", otherwise a miss has occurred and classify it as "unknown" and add this member to the face library with its feature vector. 


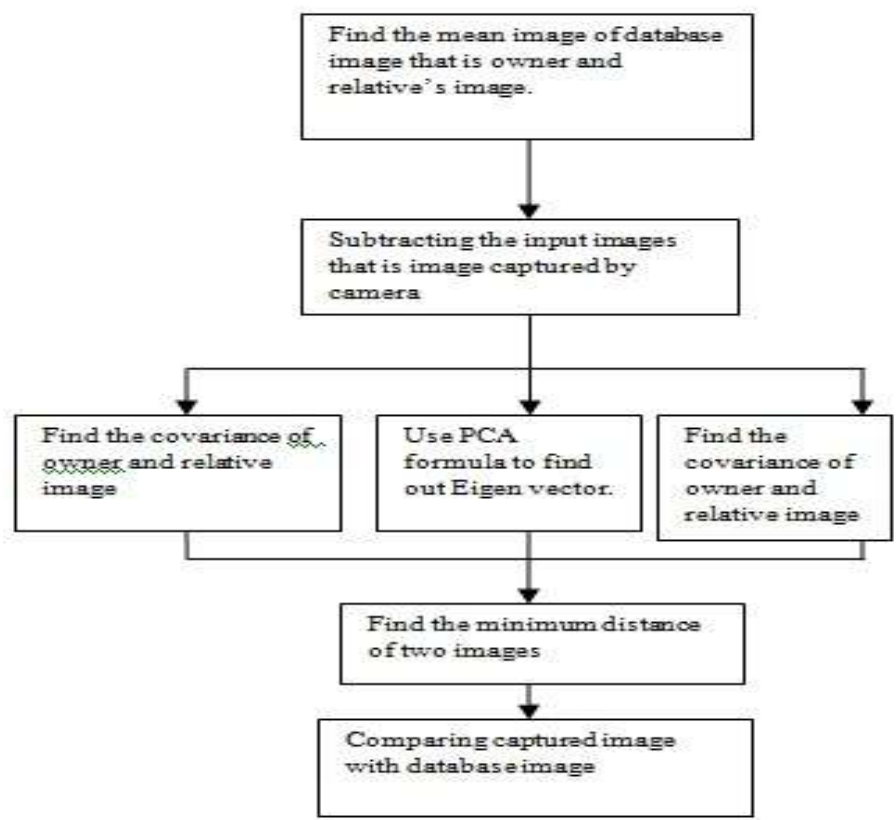

Fig.3. Face recognition based on PCA

\section{Gps Module}

GPS(Global Positioning System) technique has been widely used in military equipments and civil devices. Jupiter TU30 GPS module is used to find out the location of car in time.GPS module has a RS-232 (Recommended Standard)cable, which can be used to communicate with many other devices like microcontroller etc in Control System.It is easy to get details like longitude,latitude,speed and other information of car. Based up on the above information received from TU30 GPS module the owner of the car can identify the precise location of car.

\section{Conclusion And Future Work}

In this security implementation of car consist of Face Recognition,GPS module, Control System,MMS and SMS sending features, is to avoid car theft and protect the usage of unauthenticated users.

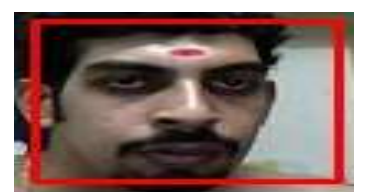

Fig4-captured image of a person at the time of Face Recognition using tiny digital camera.

Secured and safety environment system for automobile users and also key points for the investigators can easily find out the hijackers image. We can predict the theft by using this system in our day to day life, and using mobile phone car can stop.

In future in addition to MMS,SMS like to add recorded audio telephonic call that means after sending MMS from car automatically a telephonic call will go to owners mobile with already recorded audio. In future it will get its applicability in almost all security systems including organizations and automobiles.

\section{Acknowledgment}

I express my earnest and deep sense of gratitude to Mrs.D.Sujatha, Head Of the Department, Computer Science and Engineering without her valuable suggestions and guidance, preparation of this Paper wouldn't have been possible. It was a great pleasure and honor to work under her and i hope many more fruitful associations in the years to come. Her kindness and affection will remain with me forever. I also owe the gratitude due towards S.Siva Sankar Rao Lecturer, Coordinator for me. And I would like to thank my guide G.Varalakshmi and other faculty members of ATRI college, for their noteworthy suggestions and encouragement given to me in completing the paper.I would also like to thanks the members of PRC(Project Review Committee) for their help, through provoking discussions invigorating suggestions extended to me with immense care, zeal throughout the work. 


\section{References}

[1] Ahmet Bahtiyar Gul "Holistic Face recognition by Dimension reduction", May 2002.

[2] Dr.Ch.D.V.Subba Rao ,Srinivasulu Asadi,V.Saikrishna (2010) A Comparative study of Face Recognition with Principal Component Analysis and Cross-Correlation Technique. International Journal of Computer Applications Volume 10- No.8, November 2010

[3] Goldberg D.E, "Genetic algorithms in search, optimization, and machine learning" Addison-Wesley, 1989.

[4] Gonzales, R and Woods R,”Digital Image Processing, 2nd Edition Prentice-Hall Englewoodcliffs, NJ, 2002.

[5] Joseph A. O'Sullivan, Robert Pless, "Advances in Security Technologies: Imaging, Anomaly Detection, Biometric Recognition", Microwave Symposium IEEE/MTT-S International Volume, Page(s):761 - 764, 2007.

[6] PU Han-lai, LING Ming, "Performance Oriented Customization of On-Chip Memory Capacity" Journal of Applied Sciences, p. 364,2005

[7] S. Ajaz, M. Asim, M. Ozair, M. Ahmed, M. Siddiqui, Z. Mushtaq,“Autonomous Vehicle Monitoring \& Tracking System,” SCONEST 2005, pp. $1-4,2005$.

[8] Zhang Yu, "Research on High Level Model and Performance Estimation" Southeast University PHD thesis, 2007.

NITHIN KURUP U.G born on August 08th 1984.He is pursuing M.Tech (Computer Science and Engineering) specialization at Aurora's Technological and Research Institute, Hyderabad, India Affiliated to JNTU-Hyderabad . His expertise includes Mobile Computing, Computer Networks. 